\title{
Grand challenges in B cell biology
}

\section{Thomas L. Rothstein 1,2,3*}

1 Center for Oncology and Cell Biology, The Feinstein Institute for Medical Research, Manhasset, NY, USA

2 Department of Medicine, Hofstra North Shore-LIJ School of Medicine, Manhasset, NY, USA

${ }^{3}$ Department of Molecular Medicine, Hofstra North Shore-LIJ School of Medicine, Manhasset, NY, USA

*Correspondence:tr@nshs.edu

"The progress of Science consists in observing interconnections and in showing with a patient ingenuity that the events of this ever-shifting world are but examples of a few general relations, called laws. To see what is general in what is particular, and what is permanent in what is transitory, is the aim of scientific thought."A.N. Whitehead

Science, immunology, and, in particular, B cell biology, often progress through multiple series of successive accomplishments and retrenchments, mixed with new discoveries and sometimes rediscoveries. But in Whitehead's terms, we have not yet arrived at a set of immutable laws for all of B cell biology. We do, however, have coherent paradigms that have proven durably explanatory (e.g., clonal selection, serological tolerance), whereas the status of others remains uncertain (e.g., idiotypic network). And beyond laws and paradigms, we have mechanisms that successfully account for some B cell behaviors (e.g., generation of diversity versus central deletion/receptor editing/peripheral anergy). And short of mechanisms, we have mysteries (such as how activated B cells choose plasmacytic versus memory B cell differentiation, or just how peripheral checkpoints operate) whose resolution will become part of future mechanisms, paradigms, and laws.

Yet even as laws, paradigms, and mechanisms are being constructed, tested, and deconstructed, B cell modulating therapies (e.g., belimumab, epratuzumab, ocrelizumab, ofatumumab, rituximab, tocilizumab, veltuzumab) are being successfully applied in clinical situations. Thus, operating principles that oppose disease and enhance health can be discerned in the absence of a complete accounting of B cell biology. Conversely, whereas much is known about the behavior of individual cells and molecules, how this all adds up to measured anti-pathogen immune responses (meaning responses that arise appropriately, end promptly, and do not damage normal tissue) when needed, and how this all adds up to tolerance for self and for the symbiotic or passenger microorganisms therein, in real life situations, remain current topics of ongoing investigations. So we have made much progress in elucidating mechanisms and developing paradigms to describe the world of B cell biology, but we are not at the immutable law stage yet. We are still identifying new participants (e.g., Breg cells), new characteristics of old players (e.g., T3 B cells), new interactions (e.g., signaling crosstalk), and new concepts (e.g., systems biology), even as we use what we know to construct practical treatments and strategies to counteract illness for the benefit of humankind.

One thing that recent and novel B cell therapies have emphasized is the importance of B cells to various immunologically mediated diseases, most prominently including rheumatoid arthritis. This coincides with the results of experiments conducted in vitro and in murine models suggesting that B cells are integral players, and may in some cases be directors, of immune responses and disease states, via intercellular interactions that include antigen presentation to $\mathrm{T}$ cells; polarization of $\mathrm{T}$ cell differentiation; membrane expression of modulatory proteins; and secretion of cytokines and chemokines that promote immune activation, differentiation, inflammation, and/or suppression; all of which together point to a role beyond the conception of B cells as mere production facilities for the generation of immunoglobulin.

As those whose object of affection is the B cell in all its subdivision and glory continue to learn more about the B cell life story, there are a number of areas in which it appears that further advances will follow from continued effort. A non-inclusive listing is provided below, to which I hope that readers of this brief essay will add, through contributions of their own.

\section{UNDERSTAND HOW THINGS HAPPEN ON A MOLECULAR SCALE}

Despite the many molecules and biochemical reactions that have been identified, we are not at the end of discovery when it comes to the factors, adapters, enhancers, and modifiers, and the interactions in which they engage, that direct B cell behavior. Like peeling an onion, new layers become serially evident, most recently in genetic regulation produced by miRNA and by epigenetic modifications. Among the many molecular avenues that are likely to be the focus of investigational efforts in coming years will be studies designed to develop an understanding of how events take place within the intracellular milieu of the B cell. Much has been learned about immune synapses, membrane migration of signaling mediators, and protein interaction sequences and targets in the submembranous region, but beyond that, it is sometimes forgotten that the inside of a cell is colloidal and not a simple aqueous solution, where viscosity, topology, location, migration, concentration, and temperature may all affect the likelihood of specific interactions. How molecules negotiate space within the interior of the B cell, and how those peregrinations determine the molecular contacts that are made, the activation thresholds that are experienced, and the biochemical rate constants that occur, is not yet clear. A second avenue of investigation is likely to concern interactions between and among molecular signals, in which the B cell acts in a dynamic sense to integrate changes produced by extracellular events (and intracellular conditions) to construct a meaningful response. This includes the realm of molecular imprinting, in which the experience of one signal, reaction, or event alters subsequent responses to another, constituting a form of "little memory" of past events at the molecular level, and further includes the interplay of transcriptional networks that control B cell fate determination and interconversion. 


\section{ELUCIDATE CELLULAR INTERACTIONS}

Although much is known regarding key intercellular interactions involving B cells, new information continually impacts this arena, such as identification and characterization of follicular T helper cells, and evidence that B cells influence T cells. Among the many cellular avenues that are likely to be the focus of investigational efforts in coming years will be studies designed to elucidate a fuller understanding of how B cell development, differentiation, and activity is influenced and supported by stromal cell niches, new flavors of T cells, and ambient cytokines. A particular conundrum lies in the interactions and influences gathered by germinal center B cells as they move within the germinal center from dark to light zone (to the extent such zones can be defined) and interact with dendritic cells and $\mathrm{T}$ cells. Aside from evaluating how $\mathrm{B}$ cells are impacted by cellular interactions, additional study is likely to revolve around how B cells influence other cells, in particular the role that B cells play in presenting antigen to, and in stimulating the activation and differentiation of, $\mathrm{T}$ cells, as well as the role B cells play in downmodulating immune cell activity. It would not be farfetched to speculate that results of this work will lead to a more balanced view of $B$ cells and T cells as equal partners in an intercommunicating and interinfluencing pathogen responsive system.

\section{RELATE MOLECULAR EVENTS AND CELLULAR INTERACTIONS TO IMMUNOLOGICAL PHENOMENA}

The impetus to study molecular events and cellular interactions is to understand and explicate the big immunological features that we are familiar with on a macroscopic level: construction and maintenance of a serological immune system that counteracts the assault of invasive organisms (and, perhaps, self-components, such as oxidized LDL) that would do us harm, and that can (hopefully) be manipulated by vaccination or other means to enhance defenses against pathogens, cellular debris, and possibly neoplastic aberrations, while maintaining selftolerance in the midst of diversity to avoid autoreactivity and destruction of autologous tissue. In this sense it is not always clear how the individual molecular and cellular parts make the in vivo whole. Among the many avenues that are likely to be the focus of investigational efforts on immune phenomena in coming years will be studies designed to elucidate the fine regulation of B cell responses and disruptions thereof that lead to chronic states of infection and autoantibody formation. In this endeavor it is likely that additional focus on molecular and cellular "brakes" involving B cells ( such as immunosuppressive PD-1 ligands, ILT family members, IL-10, and adenosine), as opposed to elements that initiate responses, will yield important information.

\section{TRANSLATE TO/INVESTIGATE THE HUMAN CONDITION}

The overall goal of immune system study is, of course, to improve the human condition. We wish to apply all that we know to prevent, reverse or ameliorate clinical disease. In that sense it is well to remember that Mus musculus and Homo sapiens diverged evolutionarily tens of millions years ago, time enough for many immune system differences to emerge and manifest themselves. As an example, Btk mutation produces widely divergent levels of $\mathrm{B}$ cell impairment in mouse (wherein B cell numbers are reduced by about half) and human (wherein agammaglobulinemia results from a virtually complete loss of B cells). Other examples abound: CD27 marks memory B cells in human, but not in mouse; CD5 marks B1 cells in mouse, but is a poor indicator of $\mathrm{B} 1$ cells in the human system; LPS stimulates mouse B cells but not human B cells; and so on. There will always be cogent reasons to probe and perturb the rodent immune system because factors that might otherwise confuse experimental results can be controlled (by inbreeding and standardized environmental conditions) and because experiments involving genetic manipulation and adoptive transfer are technically and ethically feasible. Animal studies provide insights that are typically not available in the human condition except through genetic "accidents of nature," but they cannot completely replace the direct study of human B cell biology. It is likely that the testing and application of murine-derived paradigms on human B cells will be an important focus of future investigational efforts. Within this context, further studies will examine the newly appreciated role of B cell dysfunction within the multifactorial pathogenesis of complex diseases such as lupus and other autoimmune dyscrasias. Murine models applied to the human condition, along with tumor genomics, will assist in identifying the molecular and cellular mechanisms responsible for malignant transformation of B cells, which constitute the cell of origin for the majority of lymphocytic leukemias and lymphomas in the western world. And new antibody-based treatments for both, as well as for immunodeficiency states produced by genetic lesions and infectious conditions, will add to the current therapeutic armamentarium. It is hoped that this will include vaccination and/or passive treatment strategies to counteract HIV, hemorrhagic fever viruses, and other emerging infectious diseases.

\section{DEVELOP NEW METHODOLOGY}

A common moot discussion topic is the one that questions whether the most important element in scientific progress is new technology that fuels new ideas and hypotheses, or new ideas and hypotheses that fuel new technology. Certainly it is easy in B cell biology to point to the former. Flow cytometry provides the means to detect low level surface antigen expression; this led directly to the identification of B1 cells, and, more recently, to single cell assessment of intracellular signaling events and immunoglobulin gene expression. Two photon excitation microscopy provides the means to track lymphocyte movement in vivo; this has led, and continues to lead, to new insights concerning germinal center mechanics. Microinjection and genetic recombination have led to innumerable advances based on over- and under-expression of specific genes. It is well to remember, however, that these are not perfect tools, and, moreover, sometimes new technology becomes broadly used without a full understanding of the inherent limitations. Flow cytometry (and sorting) can be confused by inadequate doublet discrimination; two photon microscopy can penetrate only a limited depth of tissue for limited periods of time; transgenic and knock-out models are hindered by effects on expression of multiple non-targeted genes. It is not clear from whence the next technological advance will emerge, but among the many innovations that may appear in coming years could well be advances in single cell analysis of protein composition and post-translational modification, as well as algorithms to codify B cell behavior and interactions during immune 
activity resulting in promulgation of testable predictions. Most importantly, it is critical to imagine new ways of probing B cell biology that can provide new kinds of information, and then make those new ways a reality.

\section{FIND NEW MOLECULES, CELLS, AND INTERACTIONS. RELATE TO IMMUNOLOGICAL PHENOMENA. TRANSLATE TO HUMAN CONDITION. ITERATE}

Perhaps it is most important to recognize that we are nowhere near the end of discovery about B cell biology or immune system function. We may have passed the beginning of the beginning, but, to quote Winston Churchill in a very different context, "This is not the end. It is not even the beginning of the end. But it is, perhaps, the end of the beginning." Someday, we can imagine, immunodeficiencies will be completely corrected, autoimmune disease will be a thing of the past, cyclic viral pandemics will be thwarted, fatal viral diseases will be counteracted, chronic mycobacterial and worm diseases will be successfully treated, if not prevented, and transplants will be fully accepted, all through manipulation of the immune system based on investigations that are taking place right at this moment, and will, with societal support, continue into the future. We are entering a golden age in terms of accumulated raw knowledge, new discovery tools, ferment among conceptual paradigms, development of antibody reagents, and movement from bench to bedside and back again. Not to be overly Panglossian, but in terms of the basic science and clinical translation of $B$ cell biology, this would seem to be the best of all possible worlds. Now is the time to put the "pedal to the metal," to re-invigorate and re-commit our efforts, and by doing so to capitalize on all that has gone before, and to effectuate the promise that the present holds for the future. For this to occur, we must move beyond the current penurious and debilitating level of support provided by government, institutional, and foundation sources. The rosy future for which current discoveries have already produced a framework cannot come to pass without cost, but the costs incurred will more than pay for themselves in a reduction in human suffering, and an improvement in the human condition, not to mention a decline in clinical expenditures (and, for those with an entrepreneurial spirit, an increase in tangible profits to be obtained from commercialization of novel reagents and therapies). Any and all of these outcomes will inure to the benefit of society at large. Thus, there is every reason, both moral and financial, for a marked increase in governmental support for NIH and in funding for biomedical research from all available sources. The challenge for the future is simply this: to elucidate and understand the laws, paradigms, and mechanisms that direct and guide $\mathrm{B}$ cell development and function, and to improve the human condition, by using what we have learned about B cell biology to prevent and treat disease. In these endeavors it is well to remember that our most important resource lies in the aggregated intellectual talent, unbridled enthusiasm, and deep-seated motivation of those who find $B$ cells the most intriguing players on the field of immunology and cell biology.

\section{ACKNOWLEDGMENTS}

I thank members of my laboratory, past and present, who have made many important contributions to the ideas expressed in this piece. I thank the National Institutes of Health for funding these efforts over many years time. I thank Drs. Barbara Kee, Tim Manser, and Kendall Smith for critical review of the manuscript.

Received: 21 January 2011; accepted: 24 January 2011; published online: 07 February 2011.

Citation: Rothstein TL (2011) Grand challenges in B cell biology. Front. Immun. 2:2. doi: 10.3389/fimmu.2011.00002 This article was submitted to Frontiers in B Cell Biology, a specialty of Frontiers in Immunology.

Copyright $\odot 2011$ Rothstein. This is an open-access article subject to an exclusive license agreement between the authors and Frontiers Media SA, which permits unrestricted use, distribution, and reproduction in any medium, provided the original authors and source are credited. 